\title{
DOS PROBLEMAS DE PROSODIA DEL GRIEGO ANTIGUO. PRIMERA PARTE: SÍLABAS CERRADAS CON ACENTO AGUDO
}

\author{
Alejandro Abritta \\ UBA - Conicet \\ alejandroabritta@gmail.com
}

\begin{abstract}
El presente artículo es la primera parte de un estudio sobre dos problemas de la prosodia del griego antiguo vinculados a la relación entre el acento tonal del lenguaje y la estructura silábica de las palabras. Luego de un resumen de algunos conceptos básicos de prosodia del griego que son utilizados a lo largo del trabajo, se analiza el problema de las palabras con sílabas cerradas con acento agudo. La principal novedad del análisis (el problema ya ha sido encarado por otros previamente) es que el corpus de estudio es la poesía homérica, fuente antes desatendida como evidencia del acento.
\end{abstract}

prosodia / griego antiguo / acento / sílaba / Homero

\section{TWO PROBLEMS OF ANCIENT GREEK PROSODY. FIRST PART: CLOSED SYLLABLES WITH ACUTE ACCENT}

This paper is the first part of a study on two problems of Ancient Greek prosody, linked with the relation between the tonal accent of the language and the syllabic structure of words. After a sum up of some of the basic concepts of Greek prosody that are used throughout the paper, it analyzes the problem of words with closed syllables marked with acute accent. The main novelty of the analysis (the problem has been previously faced by others) is that the corpus used is the Homeric poetry, until today unattended as a source of evidence for Greek accent.

prosody / ancient greek / accent / syllable / Homer 
Introducción

E l presente trabajo intenta encarar, como indica su título, dos problemas de la prosodia del griego antiguo ${ }^{1}$. Ambos conciernen a la relación entre los distintos tipos de sílabas cerradas en la lengua y la connotación que constituye, de acuerdo a Allen (1973 y 1987), al que se seguirá aquí, su acento. El problema que se encarará en esta primera parte ya ha sido tratado por otros autores ${ }^{2}$; el otro, por razones que se harán claras inmediatamente, es por primera vez estudiado aquí (aunque ha sido observado por otros). Trabajos anteriores (en particular, los de Devine y Stephens, especialmente Devine y Stephens 1994) han encarado problemas similares a partir de un estudio de los textos con notación musical preservados, sobre la premisa metodológica de que éstos son la única fuente directa con la que contamos para un estudio del acento griego. Sin embargo, en éste, y siguiendo las investigaciones de Nagy (1996: 129-132, 2000 y 2010) y David (2006) se utilizará un criterio distinto, en la medida en que se aceptará que el acento tonal de la lengua griega cumplía un rol en la poesía griega. Por ello, el corpus que se utilizará son los poemas homéricos ${ }^{3}$, y el análisis no dependerá de una comparación entre los acentos gráficos y la notación musical sino de un estudio de la distribución de esos acentos.

El presente artículo es la primera de las dos partes de este trabajo, dada la extensión de cada una de ellas. Sin embargo, hay una continuidad indisoluble a lo largo de todo el estudio, en la medida en que se utilizan siempre las mismas premisas metodológicas y teóricas, pero también porque los resultados alcanzados en esta primera parte son tomados en cuenta en la segunda.

La primera sección del texto realiza un resumen de algunos de los principales conceptos teóricos que se utilizarán a lo largo de todo el estudio. Tras esto, se encarará el primer problema, en el orden que se utilizará en ambos: formulación, metodología, pruebas y conclusiones. Entre las primeras dos secciones he introducido aquí una revisión de la evidencia anteriormente presentada sobre el tema. Finalmente, recomiendo al lector que no esté interesado en seguir en detalle los análisis estadísticos que se realizarán pasar directamente de la formulación del problema (sec. 2.1) a las conclusiones (sec. 2.5), donde se resumen los resultados alcanzados.

A partir de este punto, "griego" quiere decir siempre "griego antiguo".

2 Cf. abajo, en las secciones 2.1 y 2.2 .

3 Más específicamente, una selección de 2484 versos de los primeros doce cantos de la llíada y los veinticuatro de la Odisea (pero con una cantidad similar de versos de cada poema). La constitución exacta del corpus, las ediciones utilizadas, los datos de este estudio y aquellos sobre los que estos se basan pueden hallarse en https://greekmps.wordpress.com. 
1. Algunas cuestiones de prosodia del griego 1.1. Estructura silábica del griego

En griego, como en otras lenguas ${ }^{4}$, existe una doble oposición al nivel del fonema y de la sílaba entre elementos largos y elementos breves. Estas oposiciones, naturalmente, se vinculan, pero no son intercambiables. En la lengua inglesa esto se hace claro en el uso terminológico: una vocal puede ser longo short, mientras que una sílaba puede ser heavy o light ${ }^{5}$. Semejante distinción no se realiza (hasta donde me consta) en español, donde tanto las vocales como las sílabas son "largas" o "breves". Es importante, no obstante, recordar la diferencia. La distinción entre sílabas largas y breves depende de una serie compleja de factores fonéticos ${ }^{6}$; la diferencia entre vocales largas y breves es fundamentalmente una cuestión de duración (una vocal breve está compuesta de una mora, una larga de dos) ${ }^{7}$.

Las vocales en griego se distinguen por el punto de articulación (que genera una variación en la frecuencia del sonido) y por la duración. Allen (1987: 62-79) ha demostrado convincentemente que el sistema está compuesto de doce sonidos representados por nueve signos ortográficos $(\alpha, \varepsilon$, $\mathrm{o}, \mathrm{t}, v, \eta, \varepsilon \mathrm{l}, \omega, \mathrm{ov})$, tres de los cuales $(\alpha, \mathrm{t}, v)$ no distinguen entre la vocal larga y la breve. La duración de la sílaba en griego (y en todas las lenguas) depende de la estructura silábica, esto es, de los componentes de la sílaba; sin embargo, la consonante inicial (o su ausencia) no tiene peso alguno en la determinación de la cantidad silábica. La presencia de una vocal larga o un diptongo determinan (siempre en términos de las formas aisladas) que la sílaba es larga; esto es independiente de la coda consonántica ${ }^{8}$. Una sílaba compuesta por una vocal breve con coda consonántica cero (esto es, por ejemplo, $\mu \breve{\alpha}$ ) constituye una sílaba breve. Finalmente, las sílabas constituidas por una vocal breve y una coda consonántica, las sílabas "cerradas", son consideradas largas.

4 Cf. Allen (1973: 43-62); Devine y Stephens (1994: 43-84, esp. 43-6).

La distinción terminológica está ya en la gramática sánscrita; cf. AlLEN (1973: 53).

Cf. Devine y Stephens (1994: 68-84), con análisis detallado del problema en griego.

Existen otros factores que las diferencian, sin embargo. La teoría del "arresto torácico" que defiende AlLEN (1973: 62-73) no ha sido suficientemente probada y la evidencia comparada (cf. Devine y Stephens 1994: 73-4, con sus referencias) sugiere que no es correcta.

8 La coda, sin embargo, permite distinguir entre sílabas largas y sílabas superlargas (DEVINE y STEPHENS 1994: 76-9); esta distinción no tiene importancia a los fines de este trabajo.

9 Técnicamente, "cerrada" se refiere a una característica de cualquier sílaba, no sólo a aquellas que tienen una vocal breve. Las sílabas superlargas son sílabas cerradas con vocal larga. Dado, sin embargo, que aquí no se considerará la diferencia entre largas y superlargas, y que sería tedioso indicar a cada paso "cerrada con vocal breve", en el presente trabajo "sílaba cerrada" quiere decir siempre "sílaba cerrada con vocal breve".

ARGOS 39 (2016) ISSN 0325-4194, pp. 49-67 
En principio, la evidencia sugiere que no había diferencias duracionales significativas entre las sílabas largas con vocal larga y las sílabas cerradas, independientemente del tipo de coda consonántica ${ }^{10}$. Esto no significa, sin embargo, que estos dos tipos de sílaba sean idénticos: en diversos idiomas en donde se da la oposición puede observarse una relación jerárquica con respecto a las preferencias de colocación del acento y al ritmo de las palabras, que pone a las sílabas cerradas un escalón por debajo de las sílabas con vocal larga ${ }^{11}$. Dentro, a su vez, de las sílabas cerradas pueden distinguirse dos grupos: las cerradas con resonante (líquidas y nasales) y las cerradas con otras consonantes ${ }^{12}$. Desde el punto de vista duracional no hay evidencia de que esta última distinción tenga algún tipo de relevancia $^{13}$, pero desde el punto de vista del acento, como se notará más abajo, no es éste el caso. El presente estudio intentará resolver dos problemas ligados a esta diferencia entre tipos de coda y, por extensión, a la relación entre el acento tonal y las sílabas cerradas.

\subsection{La contonación}

El acento griego era tonal o melódico. Esto implica que el elemento fonético prominente en su ejecución no era la intensidad o la duración, sino el tono. Si se dejan de lado los enfoques generativos ${ }^{14}$, cuyo objetivo es en general derivar a partir de la estructura rítmica de las palabras griegas la posición del acento ${ }^{15}$, la teoría de Allen de la contonación es hoy la más extendida y aceptada sobre la naturaleza del acento griego ${ }^{16}$. Ha si-

10 Cf. DeVine y Stephens (1994: 70-76).

11 Cf. Devine y Stephens (1994: 70-73), con sus numerosas referencias.

12 Adopto el término "resonante" para líquidas y nasales a partir de DEVINE y STEPHENS (1994: 191). Para referirme al resto de las consonantes usaré más adelante "oclusivas", pero debe recordarse que este grupo también incluye la sibilante $\sigma i \gamma \mu \alpha$. La terminología es inapropiada; la conjunción no lo es, porque la sibilante no muestra (en las pruebas que se realizarán aquí) un comportamiento diferente al de las oclusivas propiamente dichas (no obstante, cf. n. 42). En todo caso, en el blog mencionado en la nota 3 puede hallarse el análisis desagregado.

13 Cf. Devine y Stephens (1994: 66-7).

14 Sobre los cuales, cf. PROBERT (2006: 112-123).

15 Esto es lo que se proponen quienes han estudiado la existencia de un pie acentual en griego (Steriade1988; Sauzet1989; y Golston 1990). Sommerstein (1973) es un ejemplo de enfoque generativo que no intenta esta derivación.

16 Sobre la historia del problema cf. PROBERT (2006: 97-112). Hay antecedentes del trabajo de Allen, en particular Misteli (1868) y JAKOBSON (1971 [1937]), pero el desarrollo de las hipótesis de cada uno de estos autores parece haberse dado en forma independiente.

ARgos 39 (2016) ISSN 0325-4194, pp. 49-67 
do, por lo demás, defendida a partir del análisis de la evidencia musical ${ }^{17}$ y del análisis del testimonio antiguo ${ }^{18}$.

Allen llama "contonación" a un movimiento de ascenso y descenso en el tono de las vocales de las palabras ${ }^{19}$. Los principios de la teoría son sencillos: el acento griego abarca hasta tres moras vocálicas; en la primera de éstas se registra un ascenso en el tono de la voz, o (acento) oxítono (de acuerdo con el griego ó̧v́vovos), mientras que en la(s) siguiente(s), si las hubiera, un descenso, no sólo con respecto al tono del oxítono, sino también al tono del resto de las sílabas. Este descenso se denomina (acento) barítono (de acuerdo con el griego $\beta \alpha \rho v ́ \tau o v o \varsigma)$, y puede completarse en una vocal larga o en dos breves. Dado que el acento griego es libre ${ }^{20}$, la única regla adicional es la de limitación, que en términos de Allen puede formularse diciendo que "no más que una mora vocálica (= una vocal breve) puede seguir a la contonación"21.

Para ubicar la contonación en la palabra (probablemente con motivos didácticos o quizás para preservar la pronunciación correcta de los poemas; claramente no son razones incompatibles) los gramáticos helenísticos diseñaron el sistema de marcas acentuales que puede hallarse en cualquier edición moderna de un texto griego ${ }^{22}$. En este sistema, el agudo (') marca el oxítono, y por lo tanto que en la(s) mora(s) siguiente(s) se halla el barítono. El acento circunflejo ( ) marca que la contonación se da completa sobre una sílaba larga (sólo en el caso de las sílabas con vocal larga). El

17 Ya por el propio AlLen (1987: 118-21), pero en particular por DeVINE y STEPHENS (1991 y 1994: 172-94).

18 Cf. DaVID (2006: 52-93).

19 Cf. Allen (1973: 234).

20 Los autores mencionados en la nota 15, especialmente STERIAdE (1988) y SAuzet (1989), se encuentran con serios problemas ante este hecho. La solución de SAuZET puede hallarse en las páginas 101-105 de su trabajo; la de STERIADE (1988: 272), que es similar, depende de una clasificación de las palabras griegas en "recesivas", con sufijo derivacional acentuado y sin sufijo derivacional pero con acento fijo en la predesinencial. La clasificación es útil, pero es importante notar que la mayor parte de las lenguas de acento libre tienen reglas para determinar la posición del acento (en el español, por ejemplo, sólo puede estar en las tres últimas sílabas de la palabra - excepto en los adverbios terminados en -mente -, y los infinitivos son siempre agudos), por lo que la propuesta no cambia el hecho de que el idioma es de acento libre.

21 "Not more than one vowel-mora (= short vowel) may follow the contonation." AlLEN $\left(1987^{3}\right.$ : 124). Cf. también Allen (1973: 236-9), Sommerstein (1973: 131-2), DeVIne y Stephens (1994: 152-6) y PROBERT (2006: 60-69).

22 Cf. Allen (198730:124-130), Devine y Stephens (1994: 171-94), Nagy (1996: 127-128) y PROBERT (2006: 55-59).

Argos 39 (2016) ISSN 0325-4194, pp. 49-67 
grave ('), que el oxítono en sílaba final frente a palabra léxica se suprime o se reduce, y en general que la contonación no se realiza ${ }^{23}$.

David (2006: 52-75 y 86-93), que sigue a Allen en la interpretación de las marcas y del acento, ha notado que el testimonio antiguo sugiere que los propios griegos clasificaban las palabras en tres tipos:con $\delta \dot{\xi} v \zeta$, $\operatorname{con} \beta \alpha \rho v ́$ y con ambos. A fin de compatibilizar esta clasificación con la teoría de Allen propone una división en las palabras marcadas con agudo:

Dependiendo de sobre qué combinación de largas y breves cayera la contonación, habrían resultado diferentes efectos. Si el descenso [del tono] caía en una vocal larga, por ejemplo, la habría ocupado completa (...), mientras que el agudo precedente sólo ocupaba una mora. La duración del descenso lo habría hecho, por lo tanto, más prominente que el ascenso (...).Si la siguiente sílaba era breve, sin embargo, probablemente no habría podido recibir el descenso [completo]. El resultado habría sido un descenso gradual desde el $\partial \hat{\xi} \dot{v} \varsigma$, enfatizando de esta manera el $\partial \hat{\xi} \dot{v} \varsigma$ ("agudo") como tal: subía hasta un punto y luego se cortaba ${ }^{24}$.

De esta manera, una palabra como äv $\theta \rho \omega \pi o \varsigma$ resulta $\beta \alpha \rho \dot{v} \varsigma$ en penúltima y una como $\theta \varepsilon \dot{c} \varsigma, \grave{o} \xi \hat{v} \varsigma$ en última. Los circunflejos, sobre la base de la analogía con el védico, donde las vocales que contienen toda la contonación están dominadas fonéticamente por el descenso del tono, también son considerados, a los fines de la clasificación, como $\beta \alpha \rho v \varsigma^{25}$.

Uno de los problemas de los que se ocupará este trabajo deriva directamente de esta clasificación. Nótese que David identifica las palabras como $\beta \alpha \rho v ́$ c a partir de la presencia de una vocal larga (esto incluye, naturalmente, a los circunflejos). Poco después de esto indica que si la sílaba que sigue al agudo es cerrada también haría que el barítono fuera la parte prominente de la contonación, aunque probablemente en un grado menor dependiendo de la sonoridad de las consonantes en la coda. La pregunta,

23 En cada una de las referencias de la nota anterior (con la excepción del texto de NAGY) puede hallarse una sección que discute la naturaleza del acento grave. En general los autores coinciden, a partir de la evidencia musical, en que el grave no era equivalente a la falta de acento, sino que consistía en un agudo debilitado.

24 "Depending on which combination of longs and shorts the contonation fell upon, different effects would have resulted. If the down-glide fell on a long vowel, for example, it would have occupied the whole of it (...), whereas the preceding acute only occupied one mora. The length of the down-glide would therefore have brought it into prominence in relation to the rise (...).If the following syllable were short, however, it probably could not have taken the glide. The result would have been a step-wise descent from the $\delta \dot{\xi} \xi \zeta$, thereby emphasizing the $\delta \xi \hat{v} \zeta$ ('sharp') as such: it rose to a point and broke off." DAviD (2006: 63).

25 Nótese que son probablemente, sin embargo, los que los griegos clasificaban como teniendo ambos acentos. La analogía con el védico es importante en el trabajo de Allen (1973: 233-4 y 1987: 121-2).

ARGos 39 (2016) ISSN 0325-4194, pp. 49-67 
naturalmente, es en qué grado se percibía esta diferencia, y si la propuesta de David puede verificarse como correcta con respecto a la clasificación de todas las palabras de este grupo.

El segundo problema es de una naturaleza diferente, aunque también concierne directamente a la relación entre la sonoridad de las consonantes, la contonación y la clasificación de las palabras. Dado que será tratado a continuación, su formulación será realizada al comienzo de la siguiente sección.

\subsection{Resumen}

La primera sección de este trabajo se dedicó a resumir algunas cuestiones básicas de prosodia del griego que deben tomarse en cuenta para entender el origen de los problemas que serán tratados en lo que sigue. En primer lugar, debe notarse especialmente las oposiciones vinculadas pero no idénticas entre vocales breves y largas y sílabas breves y largas. La categoría de sílaba cerrada, esto es, las sílabas largas con vocales breves, será la que estará en el centro de las discusiones en las siguientes secciones. En segundo lugar, es importante que el lector se familiarice con los

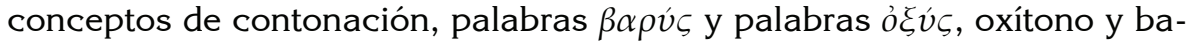
rítono, porque serán utilizados con gran frecuencia en el tratamiento de los problemas.

Este trabajo utilizará una triple clasificación con respecto al acento griego. Primero, se apelará al sistema tradicional de categorización de las palabras (oxítonas, paroxítonas, proparoxítonas, perispómenas y properispómenas) para referirse a la posición de las marcas acentuales. Segundo, se utilizará "barítono" y "oxítono" (nótese que ambos son masculinos) para indicar las partes de la contonación y su posición en la palabra ${ }^{26}$. Finalmente, $\beta \alpha \rho \dot{v} \varsigma$ y óǵv́ se usarán siguiendo el esquema de David (2006), descrito en la sección anterior.

${ }^{26}$ Es importante distinguir entre "oxítona" y "oxítono". El primer término se refiere a la posición de un tipo de marca acentual (el agudo) en una palabra, el segundo a una de las partes de la contonación que constituye el acento. Algunas palabras griegas son oxítonas, pero todas (las léxicas) tienen oxítono.

Utilizaré en este trabajo otra distinción terminológica, en este caso por una cuestión práctica, no teórica. La palabra "posición" se referirá en todos los casos a una sílaba o una parte de una palabra, y por lo tanto se utilizará primordialmente para hablar del lugar del acento en un lexema; la palabra "ubicación" se referirá en todos los casos a una sílaba o una parte del hexámetro. Así, "penúltima sílaba" es una posición, y "tercera tesis" una ubicación.

ARgOS 39 (2016) ISSN 0325-4194, pp. 49-67 
2. Sílabas cerradas con acento agudo

\subsection{Formulación del problema}

La contonación inicia en una mora y sigue en la(s) siguiente(s). Cuando comienza en una sílaba larga, el circunflejo indica que el ascenso del tono está en la primera mora, y el descenso en la segunda, mientras que el agudo indica que el ascenso del tono está en la segunda mora. Cuando la vocal es larga, esto no presenta inconveniente alguno, sea cual fuere la posición de la sílaba en la palabra. Sin embargo, cuando la vocal es breve, no hay en realidad primera y segunda mora (al menos vocálicas), como indica claramente la imposibilidad de colocar circunflejos sobre este tipo de sílaba. La pregunta entonces es cómo deben interpretarse los agudos sobre sílabas cerradas.

Cuando a la sílaba sigue otra larga, no hay problema alguno, puesto que la contonación se completa en ésta ${ }^{27}$. El inconveniente surge cuando la sílaba cerrada está en final de palabra en sandhi (no hay otra manera de que haya una sílaba cerrada en final de palabra $)^{28}$ y, sobretodo, cuando a la cerrada siguen una o más vocales breves ${ }^{29}$. Dada la regla de limitación, cuando la sílaba es la antepenúltima en una configuración dactílica podemos inferir a priori que se comporta en todos los casos como un agudo sobre vocal larga ${ }^{30}$. El problema se da en los casos donde una sílaba cerrada con agudo está en penúltima posición (o en última en sand$h i$, pero por mor de la simplicidad se pueden derivar las reglas para estos casos de las que se obtengan para los otros) en palabras de final trocaico. Para David (2006: 65), en estos casos, la palabra es $\beta \alpha \rho v ́ c$ en penúltima:

La regla que debemos proponer es que la contonación puede empezar y ser completada no sólo en una vocal larga, sino también en una sílaba cerrada con vocal breve, provisto que no haya más de una mora siguiendo a la compleción.

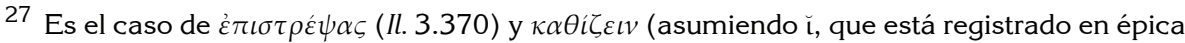
según LSJ Il. 3.394).

28 "Sandhi" es el término sánscrito, hoy adoptado en lingüística, para referirse a los cambios fonéticos que las palabras sufren en determinados contextos fonológicos, es decir, en su contacto con otras.

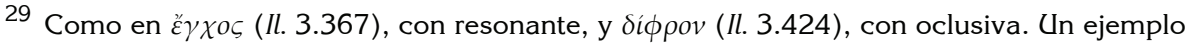

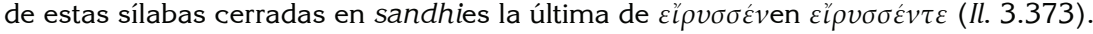

30 Como en ع̌ $\sigma \pi \varepsilon \tau o$ (Il. 3.376).No me ocuparé aquí de probar esta hipótesis, pero una prueba es posible. En todo caso, resulta difícil creer que una configuración tan frecuente viole una regla tan fundamental del acento griego.

ARGos 39 (2016) ISSN 0325-4194, pp. 49-67 
Sin embargo, este fenómeno, conocido ya por Vendryes (1904, §92) y el propio Allen (1987: 127, n. 31) es interpretado en forma diferente por estos autores. Para ellos, esta regla para las sílabas cerradas en penúltima de un troqueo es válida sólo en el caso de que la coda consonántica tenga una resonante. En estos casos, la contonación se completa en la consonante, que daría a la sílaba una similitud grande con las palabras perispómenas. El problema que será tratado en esta sección es cuál de las tres interpretaciones posibles de estas sílabas cerradas con agudo es correcta:

1. La interpretación estricta basada en las reglas de la contonación que las entiende como simples agudos sobre vocal breve.

2. La interpretación de David que entiende que en todos los casos deben

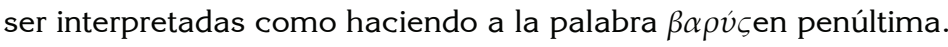

3. La interpretación de Vendryes y Allen que entiende que sólo cuando la coda consonántica es una resonante deben ser interpretadas como haciendo a la palabra $\beta \alpha \rho v ́ \varsigma e n$ penúltima.

\subsection{Evidencia en papiros y textos musicales}

Antes de realizar pruebas originales, puede resultar útil repasar la evidencia que otros autores han producido sobre el tema. Allen y Vendryes fundamentan su posición en la ortografía de los papiros y los manuscritos,

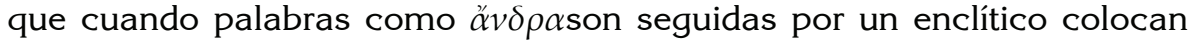

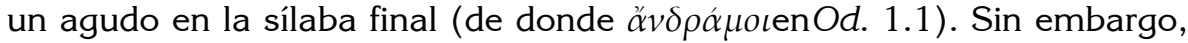
los mismos autores critican a los gramáticos antiguos (que establecieron la regla para los papiros y manuscritos) porque extendieron esta notación a todas las sílabas cerradas (de donde ö $\phi \rho \alpha ́ \mu \iota \iota$ en Il. 5.110). La evidencia ortográfica, por esto, apoya la posición de David, y se requiere postular que los gramáticos estaban equivocados en la notación que proponen para sostener la de Allen y Vendryes.

Más interesante, sin embargo, resulta el análisis de los textos con notación musical que realizan Devine y Stephens (1994: 191-4). Aunque las conclusiones que obtienen los autores no son contundentes, la preferencia por realizar melismas en sílabas cerradas con resonante sobre realizar melismas en cerradas con oclusiva es significativa en el caso de los melismas pre-acentuales, con agudo y post-agudos (pero sólo en dos de estos tres casos estadísticamente significativa). Esto sugiere que la posición de Allen y Vendryes tiene fundamento en la evidencia. 


\subsection{Metodología}

Es bien sabido que la distribución de palabras en el hexámetro homérico no es arbitraria, sino que sigue reglas, a veces estrictas ${ }^{31}$. Menos evidente es el hecho de que a estas tendencias de métrica interna se añaden otras vinculadas a las preferencias en la colocación de los acentos ${ }^{32}$. Siendo esto así, debería ser posible utilizar estas preferencias para identificar si un tipo de palabra en particular corresponde a una categoría acentual determinada o no. Provisto que alguna de estas preferencias involucrara palabras trocaicas, y sobretodo opusiera palabras $\beta \alpha \rho v ́ \varsigma$ en penúltima

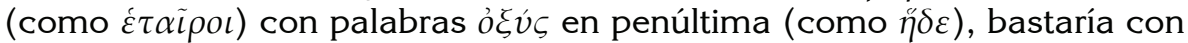
confrontar la distribución de los dos tipos posibles de palabras con sílaba cerrada en penúltima con la de las palabras con vocal larga en penúltima a los fines de determinar qué tipo de acento tienen esta palabras.

El primer paso es, entonces, ubicar, a partir de un análisis de la distribución de palabras trocaicas con vocal larga en penúltima, una posición del metro que exhiba una preferencia clara por o contra palabras $\beta \alpha \rho v ́$ ऽ o palabras $\delta \xi \xi \hat{v} \varsigma$. El segundo, analizar la distribución de las palabras trocaicas con sílaba cerrada en penúltima sin distinguir tipos de estas palabras, a fin de corroborar si existen preferencias que sean independientes del acento (este paso es necesario para aislar variables que puedan estar afectando las pruebas definitorias). Finalmente, debe conducirse un análisis de la distribución de los diferentes tipos de sílabas cerradas, comparándola con la de las palabras con vocal larga, a fin de confirmar a qué tipo de acento cada tipo de sílaba cerrada se asocia.

\subsection{Prueba}

\subsubsection{Distribución de las palabras de final trocaico con penúltima larga}

A fin de no generar distorsiones, el análisis excluye enclíticos y proclíticos trocaicos (como $\dot{\varepsilon} \sigma \tau \iota$ y $\dot{\alpha} \lambda \lambda \dot{\alpha}$ ), así como palabras métricas (o grupos apositivos) constituidos por una ortotónica con sílaba final larga y un

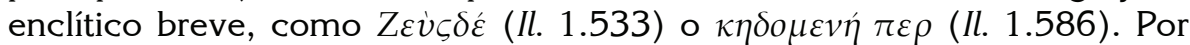
otro lado, las palabras con final elidido han sido incluidas, provisto que la forma de hecho en la que aparecen en el verso tenga final trocaico (e.g.

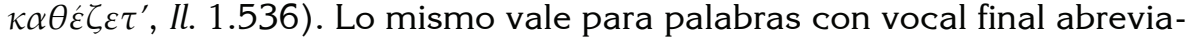

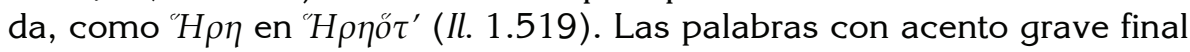

31 Cf. O'Neill (1942), Porter (1951) y BeEkes (1972).

32 Cf. ABritTA (2010). Me encuentro preparando un trabajo que demuestra más claramente este hecho. 
han sido incluidas en el grupo de las oxítonas, pero removerlas no altera los resultados ${ }^{33}$. Los resultados se muestran en la tabla 2.1.

\begin{tabular}{|c|c|c|c|c|c|c|}
\hline Pal. Tr. (V.L.) & $1^{\circ}$ pie & $2^{\circ}$ pie & 3ㅇ pie & 5우 pie & $6^{\circ}$ pie & Total \\
\hline Oxítonas & $54(22,69 \%)$ & $15(13,39 \%)$ & $76(9,84 \%)$ & $\begin{array}{r}93 \\
(17,92 \%)\end{array}$ & $71(9,22 \%)$ & $\begin{array}{r}309 \\
(12,82 \%)\end{array}$ \\
\hline en pen. & $48(20,17 \%)$ & $\begin{array}{r}17 \\
(15,18 \%)\end{array}$ & $27(3,50 \%)$ & $\begin{array}{r}54 \\
(10,40 \%)\end{array}$ & $1(0,13 \%)$ & $\begin{array}{r}147 \\
(6,10 \%)\end{array}$ \\
\hline en pen. & $136(57,14 \%)$ & $80(71,43 \%)$ & $\begin{array}{r}669 \\
(86,66 \%) \\
\end{array}$ & $\begin{array}{r}372 \\
(71,68 \%) \\
\end{array}$ & $\begin{array}{r}698 \\
(90,65 \%) \\
\end{array}$ & $\begin{array}{r}1955 \\
(81,09 \%) \\
\end{array}$ \\
\hline Total & 238 & 112 & 772 & 519 & 770 & 2411 \\
\hline
\end{tabular}

Tabla 2.1. Distribución de distintos tipos de palabras de final trocaico con vocal larga en el hexámetro de Homero; se muestran los porcentajes por columna entre paréntesis.

A fin de facilitar la interpretación de los resultados, en cada celda se incluye el porcentaje de cada tipo de acento por columna, incluyendo las del total. La tabla presenta los tres tipos posibles de palabras de final trocaico, las oxítonas (ỏ $\hat{v} v$ en última, de acuerdo a la clasificación de David),

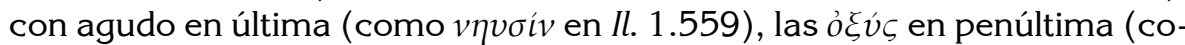
mo $\pi \tau \dot{v} \gamma \mu \alpha$ en Il. 5.315) y las $\beta \alpha \rho \dot{v} \varsigma$ en penúltima (como $\alpha \alpha \tau \alpha \dot{v} \varepsilon v \sigma o v$ en Il. 5.514 y $\chi \varepsilon i \tilde{\rho} \alpha \varsigma$ en Il. 1.567). Las columnas indican el pie donde la palabra termina, entendiendo que la penúltima se ubica en la tesis o princeps de ese pie y la última en la primera posición del arsis o bíceps (la única ubicación posible para palabras trocaicas en el hexámetro). En el sexto pie he dejado de lado el principio brevis in longo, entendiendo que, aunque permite la ubicación de palabras tanto trocaicas como espondaicas, no neutraliza la diferencia entre ambas ${ }^{34}$. He excluido el cuarto pie, dado que las palabras trocaicas allí están limitadas por el puente de Hermann ${ }^{35}$. Por otro lado, tomando en cuenta que Cantilena (1995) ha probado que la prime-

33 Puede corroborarse en [link omitido por mor del anonimato], donde las pruebas sin los graves son presentadas en un archivo aparte.

34 Espero publicar prontamente un trabajo que demuestre que éste es el caso. Por lo pronto, baste con señalar que es lo que se extrae de las interpretaciones más generalizadas del principio (cf. MAAS 1962: §34, WeST 1982: 4 y en particular SILVA-BARRIS 2011: 38-9).

35 El puente de Hermann (MAAS, 1962: §87; WEST, 1982: 37-8) limita la ubicación de palabras de final trocaico en el arsis del cuarto pie del hexámetro. Hay, de hecho, solamente 33 palabras de final trocaico con vocal larga en esta posición en el corpus. De éstas, 28 son $\beta \alpha \rho v ́ \varsigma$ en penúltima (como $\Pi v \theta \tilde{\omega} v \alpha ́$ en Il. 2.519, seguida por $\tau \varepsilon$ y por lo tanto no violando el Puente de Hermann), 21 de las cuales tienen un barítono prominente post-agudo (como $\varepsilon \check{\lambda} \omega \sigma i$ en Il. 4.416, también seguido de $\tau \varepsilon$ ). 
ra ley de Meyer no se aplica en Homero, no he excluido el segundo pie $^{36}$. Nótese, sin embargo, que es la posición con menor cantidad de palabras de final trocaico (lo que es consistente con los resultados de Cantilena).

Aunque hay muchos datos interesantes que pueden obtenerse a partir de esta tabla, a los fines del presente trabajo es evidente que el más importante es el que se halla en la celda correspondiente a las palabras $\delta \hat{\xi} \dot{v} \zeta$ en penúltima en el 6우 pie. En esa ubicación se encuentra el único porcentaje inferior al $1 \%$, en una posición particularmente significativa ${ }^{37}$, porque es la segunda que mayor cantidad de palabras trocaicas tiene (con el 36\% del total). Algo similar sucede en el tercer pie, pero la tendencia es bastante menos marcada.

La evasión de $\grave{o} \xi \hat{v} \zeta$ en penúltima en el 6 o pie será por lo tanto el criterio de juicio para descubrir cuál era la prosodia de las sílabas cerradas de final trocaico con agudo en penúltima.

\subsubsection{Distribución de palabras de final trocaico con sílabas cerradas}

Aunque Devine y Stephens (1994: 59-84) ya han demostrado que las diferencias entre las sílabas largas con vocal larga y con vocal cerrada no tienen valor métrico ${ }^{38}$, resulta importante repetir la prueba aquí porque el análisis de estos autores se realizó sobre una muestra de trímetro y porque parece sensato no escatimar en precauciones. Los resultados que se muestran en la tabla 2.2 respetan las mismas limitaciones que las descritas para la tabla 2.1. Los porcentajes entre paréntesis en este caso, sin embargo, corresponden al total por fila (no por columna), a fin de comparar si la distribución de los dos tipos de sílabas largas es similar a lo largo del hexámetro.

36 Sobre el problema de la ley de Meyer, que limita la ubicación de palabras de final trocaico en el segundo pie del hexámetro, además del detallado trabajo de Cantilena, cf. BECK (1972) y BAKKER (1986).

37 La única palabra de este tipo en todo el corpus de 2484 versos (esto es, el único final de verso de este tipo en 2484 hexámetros) es oíde en Od. 4.235, en un encabalgamiento violento, en sí mismo un fenómeno infrecuente en Homero.

38 Más específicamente, que su valor métrico está limitado a ciertos contextos muy específicos. La explicación rítmica que los autores dan de este fenómeno no afecta las conclusiones del presente estudio. De hecho, es plausible considerar que una revisión de sus análisis debe ser realizada desde una perspectiva que tome en cuenta el acento de las palabras.

ARGos 39 (2016) ISSN 0325-4194, pp. 49-67 


\begin{tabular}{|c|c|c|c|c|c|c|}
\hline Pal. Tro. & $1^{\circ}$ pie & $2^{\circ}$ pie & 3으 pie & 5우 pie & $6^{\circ}$ pie & Total \\
\hline Vocal larga & $238(9,87 \%)$ & $\begin{array}{r}112 \\
(4,64 \%)\end{array}$ & $\begin{array}{r}772 \\
(32,01 \%)\end{array}$ & $\begin{array}{r}519 \\
(21,52 \%)\end{array}$ & $\begin{array}{r}770 \\
(31,97 \%)\end{array}$ & 2411 \\
\hline Cerradas & $\begin{array}{r}147 \\
(10,92 \%)\end{array}$ & $57(4,23 \%)$ & $\begin{array}{r}391 \\
(29,05 \%)\end{array}$ & $\begin{array}{r}325 \\
(24,15 \%)\end{array}$ & $\begin{array}{r}426 \\
(31,65 \%)\end{array}$ & 1346 \\
\hline Total & $\begin{array}{r}385 \\
(10,24 \%)\end{array}$ & $\begin{array}{r}169 \\
(4,50 \%)\end{array}$ & $\begin{array}{r}1163 \\
(30,95 \%)\end{array}$ & $\begin{array}{r}844 \\
(22,46 \%)\end{array}$ & $\begin{array}{r}1196 \\
(31,85 \%)\end{array}$ & 3757 \\
\hline
\end{tabular}

Tabla 2.2. Distribución de palabras de final trocaicocon distintas estructuras silábicas en el hexámetro de Homero; se muestran los porcentajes por fila entre paréntesis.

A simple vista los resultados parecen demostrar que no hay diferencia alguna en ninguna ubicación del metro entre las vocales largas y las sílabas cerradas. Una prueba de $\chi^{2}$ confirma esto, ya que la posibilidad de que los números de esta tabla estén distribuidos aleatoriamente (dados los totales) es del $17 \%(\chi=6,38)^{39}$. Esto significa, sencillamente, que el poeta no exhibe preferencia alguna por ninguna estructura silábica en ninguna ubicación; coloca en cada una indiferentemente cualquier sílaba larga, por lo menos en lo que respecta a las palabras de final trocaico.

Demostrado esto, si se hallaran diferencias en la distribución en distintos tipos de sílabas cerradas con acento agudo, éstas deberían atribuirse con toda probabilidad a la preferencia por cierto tipo de acento ${ }^{40}$.

39 Los resultados desagregados por tipo de sílaba cerrada, así como los de los disílabos trocaicos parecen no estar distribuidos aleatoriamente, dados los resultados de una prueba de ${ }^{2}$, lo que iría en contra de los resultados de DeVINE y STEPHENS y de un criterio exclusivamente acentual para la distribución de las palabras. Un análisis más detenido del problema (pueden consultarse las tablas en [link omitido por mor del anonimato]) demuestra que los resultados no son particularmente graves para los objetivos del presente estudio. En el caso del análisis desagregado por tipos de sílaba cerrada, ambos están siempre en la misma dirección con respecto a las cantidades esperadas, lo que sugiere que si hay alguna preferencia, no hace diferencias entre oclusivas y resonantes, por lo que una diferencia en la distribución de estos grupos sólo puede ser explicada desde el punto de vista del acento. En el caso de los disílabos trocaicos el problema es algo más complejo, porque las resonantes y las oclusivas se comportan en forma opuesta en varias ubicaciones. En este caso, sin embargo, dada la exclusión de los proparoxítonos, en donde no parece haber diferencia prosódica entre ambos grupos (cf. la segunda parte de este trabajo, sec. 3.1.1), queda a criterio del investigador si la diferencia es métrica (lo que resulta realmente improbable) o acentual. En todo caso, es importante notar que, dado que los resultados agregados muestran una probabilidad muy alta de distribución aleatoria, seguir el camino de las diferencias métricas no parece la mejor estrategia.

40 Existe, por supuesto, la posibilidad teórica de que haya algún tipo de diferencia exclusivamente métrica entre los diferentes tipos de sílaba cerrada (sin embargo, cf. la

ARgos 39 (2016) ISSN 0325-4194, pp. 49-67 


\subsubsection{Distribución de palabras con final trocaico, por estructura silábica $y$ acento}

La manera más directa de realizar la prueba que se necesita es revisar la cantidad y el tipo de acento de las palabras con final trocaico con sílaba cerrada por oclusiva y resonante. Debe notarse que, dado que de momento no se están problematizando las palabras con barítono post-agudo, este grupo será excluido de los análisis que siguen (en el caso de las sílabas cerradas, por lo menos) ${ }^{41}$. En la tabla 2.3 se hallan las cantidades absolutas desagregadas por tipo.

Es necesario ahora comparar estas distribuciones con las de la tabla 2.1, que muestran las de las palabras con vocal larga en penúltima. Esto no resulta tan sencillo como podría pensarse a primera vista, porque no se puede simplemente oponer porcentajes: como resulta obvio al estudiar las estructuras de las tablas, la primera tiene tres filas que muestra tres categorías acentuales diferentes y la segunda cuatro que muestra dos categorías acentuales de dos estructuras silábicas. Ahora bien, dado que los porcentajes provienen de los totales, y los totales provienen de cantidades y combinaciones distintas de categorías, se debería saber de antemano qué categorías deben compararse y cuáles no, y eso es precisamente lo que se intenta averiguar.

\begin{tabular}{|c|c|c|c|c|c|c|}
\hline Pal. Tr. (S. C.) & $1^{\circ}$ p pie & $2^{\circ}$ pie & 3ㅇp pie & 5ㅇ pie & $6^{\circ}$ pie & Total \\
\hline Oc.: Oxítonas & 13 & 5 & 18 & 29 & 24 & 89 \\
\hline Oc.: Ag. Penúl. & 49 & 17 & 49 & 49 & 17 & 181 \\
\hline Re.: Oxítonas & 18 & 0 & 23 & 47 & 10 & 98 \\
\hline Re.:Ag. Penúl. & 67 & 67 & 67 & 67 & 67 & 335 \\
\hline Total & 147 & 89 & 157 & 192 & 118 & 703 \\
\hline
\end{tabular}

Tabla 2.3. Distribución de palabras de final trocaicocon distintas estructuras silábicas ("Oc." = cerrada por oclusiva en penúltima; "Re." = cerrada por resonante en penúltima) y distintos tipos de acento en el hexámetro de Homero.

nota anterior), pero es con toda seguridad la interpretación más inverosímil de un resultado que muestre una distribución con ciertas preferencias.

41 En el caso de las sílabas con vocal larga, se podría, por precaución, tomar la misma medida, y dejar únicamente los circunflejos en el grupo de las palabras $\beta \alpha \rho v ́$ s en penúltima (que, nótese de paso, son los únicos $\beta \alpha \rho v ́$ c trocaicos posibles). Sin embargo, no hay diferencia significativa entre estos acentos y los barítonos post-agudo (esto puede verificarse en el blog mencionado en la n. 3) que justifique realizar este recorte. 
Este problema surge precisamente del que se está intentando resolver: todas las interpretaciones posibles de las sílabas cerradas con agudo excluyen uno de los dos grupos de acento en penúltima de la tabla 2.1. Si la interpretación básica fuera correcta, entonces todos los agudos en penúltima en sílaba cerrada serían ó̉v́c, y las dos filas "Ag. Penúl." en la tabla 2.3 estarían contando la misma categoría acentual que "Oگ̌́s en pen." en la tabla 2.1; si la de David, todos serían $\beta \alpha \rho v ́ \varsigma, y$ las dos filas "Ag. Penúl."

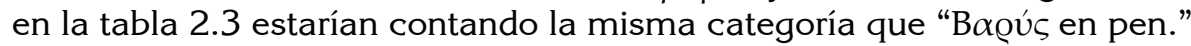
en la tabla 2.1; si la de Allen y Vendryes, el primer par (las oclusivas) sería ỏ̉v́c y el segundo (las resonantes) sería $\beta \alpha \rho \dot{v} \varsigma$, por lo que "Oc.: Ag. Penúl." en la tabla 2.3 estaría contando la misma categoría que "Očús en pen." en la tabla 2.1, mientras que "Re.: Ag. Penúl." estaría contando la

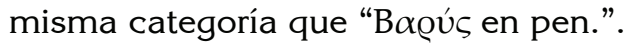

Ahora bien, una medida que puede utilizarse sin necesidad de establecer subgrupos en la tabla 2.1 es la razón entre diferentes celdas de cada columna, esto es, cuántas palabras de un grupo hay por cada palabra del otro. En la tabla 2.4, estas medidas se muestran para cada combinación relevante de la tabla 2.1 (se excluye la razón $\delta \xi \dot{v} \varsigma / \beta \alpha \rho v ́ c$, porque no hay con qué compararla) y para cada par de la tabla 2.3.

\begin{tabular}{|c|c|c|c|c|c|c|}
\hline Razones & 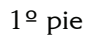 & $2^{\circ}$ pie & $3^{\circ}$ pie & 5으 pie & 6o pie & Total \\
\hline V.L.: . / Ox. & 0,89 & 1,13 & 0,36 & 0,58 & 0,01 & 0,48 \\
\hline V.L.: . / Ox. & 2,52 & 5,33 & 8,80 & 4,00 & 9,83 & 6,33 \\
\hline Oc.: Ag. / Ox. & 3,77 & 3,40 & 2,72 & 1,69 & 0,71 & 2,03 \\
\hline Re.: Ag. / Ox. & 3,72 & - & 2,91 & 1,43 & 6,70 & 3,42 \\
\hline
\end{tabular}

Tabla 2.4. Razones entre cantidades de palabras con diferentes tipos o marcas

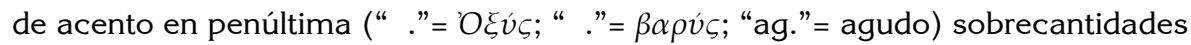
de palabras con acento en última sílaba ("Ox.") en palabras con final trocaico con diferentes estructuras silábicas ("V. L." = vocal larga en penúltima; "Oc."= oclusiva en penúltima; "Re."= resonante en penúltima) en el hexámetro de Homero. El guión indica que la razón no se puede obtener porque uno de los números de la división es 0 .

Los datos de esta tabla no son tan complejos como podría pensarse. Cada celda indica simplemente qué cantidad de palabras del dividendo hay por cada palabra del divisor. La primera de la izquierda, por ejemplo, indica que, en el primer pie del hexámetro,hay 0,89 palabras de final tro-

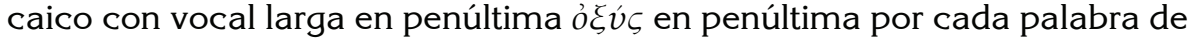


final trocaico con vocal larga en penúltima oxítona; la última de la derecha (exceptuando la columna con los totales) que, en el sexto pie del hexámetro, hay 6,7 palabras de final trocaico con sílaba cerrada por resonante en penúltima y acento agudo en penúltima por cada palabra de final trocaico con sílaba cerrada por resonante en penúltima oxítona. En otras palabras, la primera celda de la izquierda indica que hay un poco más de oxítonas

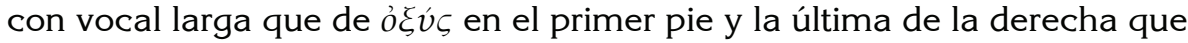
hay muchas más cerradas por resonante con agudo en penúltima que oxítonas en el sexto.

Un fenómeno que salta a la vista rápidamente es que los números de los primeros cinco pies de las palabras con los dos tipos de sílaba cerrada son muy similares entre $\mathrm{si}^{42}$ y distintos a los de las palabras con vocal lar$\mathrm{ga}^{43}$. Sin embargo, en el sexto pie, que es la ubicación clave del análisis (cf. sec. 2.4.1.) la diferencia es grande entre todas las celdas de la columna. Dicho esto, parece claro que hay en ella dos grupos bien identificables: tanto

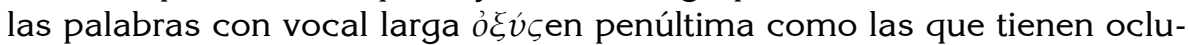

\footnotetext{
$\overline{42}$ Este resultado es, sin embargo, algo engañoso. Obsérvese lo que sucede si se toman por separado (cf. n. 12) las palabras con sílaba cerrada con sibilante y con oclusiva:

Como puede verse, los números son diferentes en casi todas las ubicaciones menos la última. Sin embargo, dado que, a los fines del presente trabajo, ésta es la que importa, sigue sin ser necesario analizar estos dos grupos por separado.

43 Este resultado no es engañoso (cf. la nota anterior), pero se relativiza bastante si se excluyen los barítonos post-agudo en las palabras con vocal larga. En particular en el sexto pie el valor de la razón baja de 6,33 a 3,26.
} 
siva con agudo en penúltima aparecen menos que las oxítonas, mientras que las palabras con vocal larga con $\beta \alpha \rho v ́$ cen penúltima y las que tienen resonante con agudo en penúltima son preferidas en esta ubicación a las oxítonas. En otras palabras, pudiendo elegir entre los opuestosprosódicos

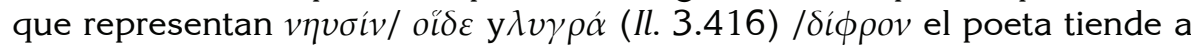

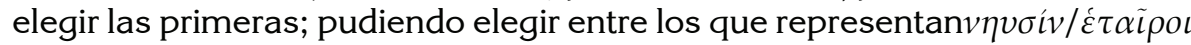
(Il. 3.378) у $\chi \varepsilon \rho \sigma i v$ (Il. 11.184) / $\check{\varepsilon} \gamma \chi 0 \varsigma$, el poeta tiende a elegir las segundas $^{44}$. Más específicamente, la evasión ya observada (sec. 2.1) de palabras $\grave{o} \xi \hat{v} \varsigma$ en penúltima (oí $\delta \varepsilon$ ) en el cierre de la línea se extiende a las palabras con sílaba cerrada por oclusiva con agudo en penúltima ( $\delta i \phi \rho o v)$, mien-

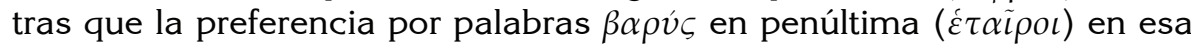
ubicación se extiende a las palabras con sílaba cerrada por resonante con agudo en penúltima ( $\left.\varepsilon^{\prime} \gamma \chi \circ \varsigma\right)$.

\subsection{Conclusiones}

Los análisis realizados apoyan la interpretación de Vendryes y Allen de la prosodia de las sílabas cerradas con acento agudo. Los resultados muestran que las palabras de final trocaico con sílaba cerrada por resonante con acento agudo en el sexto pie del hexámetro se comportan como $\beta \alpha \rho v ́ s$ en penúltima, asimilándose marcadamente a los circunflejos y los barítonos post-agudo. Por otro lado, aunque se observa una cierta evasión de palabras de final trocaico con sílaba cerrada por oclusiva con acento agudo, es claro que no es tan marcada como en el caso de las palabras $\dot{o} \xi \hat{v} \zeta$ en penúltima (de las cuales, nuevamente, hay un solo caso en casi dos mil quinientos versos). Esto, sumado al hecho de que en el resto de la línea no parece haber grandes diferencias entre los distintos tipos de sílabas cerradas, sugiere que las palabras con agudo en sílaba cerrada por oclusiva se pueden asimilar a las $j \xi \hat{v} v$ en penúltima con vocal larga, pero con la aclaración de que su prosodia probablemente se percibía como intermedia entre este grupo y el de las sílabas cerradas por resonante.

$\overline{44}$ Nótese que esto es una tendencia, no una regla absoluta (de hecho, todos los ejemplos fueron tomados del texto).

ARGOS 39 (2016) ISSN 0325-4194, pp. 49-67 


\section{Bibliografía}

ABritTA, A. (2010) "Sobre la posibilidad de un análisis coral en Ilíada 53305", Anales de Filología Clásica,23, pp. 1-62.

Allen, W. S. (1973) Accent and Rhythm, Cambridge: University Press.

Allen, W. S. (1987) VoxGraeca, Cambridge: University Press.

BAKKER, E. J. (1990) "Homeric Discourse and Enjambement: A Cognitive Approach", TAPhA, 120, pp. 1-21.

Beck, R. (1972) "A Principle of Composition in Homeric Verse", Phoenix,26, pp. 213-231.

BEEkES, R. S. P. (1972) 'On the Structure of the Greek Hexameter: 'O'Neill' Interpreted", Glotta,50, pp. 1-10.

Cantinela, M. (1995), "Il ponte di Nicanore",en M. Fantuzzi, R. Pretagostini (eds.) Struttura e storiadell'esametro greco, vol. I, Roma: Gruppo Editorial e Internazionale, pp. 9-68.

David, A. P. (2006) The Dance of the Muses. Choral Theory and Ancient Greek Poetics, Oxford: University Press.

Devine, A. M. y Stephens, L. D. (1991) "Dionysius of Halicarnassus, De CompositioneVerborum XI: Reconstructing the Phonetics of the Greek Accent", TAPhA, 121, 229-286.

Devine, A.M. y Stephens, L. D. (1994) The Prosody of Greek Speech. New York and Oxford: Oxford University Press.

Golston, C. (1990)"Floating H (and L*) Tones in Ancient Greek",en J. Myers y P. E. Pérez (eds.), Arizona Phonology Conference III,Tucson: University of Arizona Linguistics Department, pp. 66-82.

Jakobson, R. (1971) "On Ancient Greek Prosody", en R. Jakobson, Selected Writings, La Haya: Mouton, 262-71 [Publicación original en polaco, 1937, "Z zagadnie prozodjistarogreckiej", en PraceofiarowaneKazimierzowiWóycickiemu(Z zagadnie poetyki,6) 73-88].

MAAS, P. (1962) Greek Metre, trans. H. Lloyd-Jones, Oxford: Clarendon Press [publicación original en alemán de 1923, Griechische Metrik, Einleitung in die Altertumswissenschaft,7, Leipzig: Teubner].

Mistel, F. (1868) "Über die Accentuation des Griechischen", Zeitschrift fürvergleichende Sprachforschung,17, pp.81-134, 161-94.

Nagy, G. (1996) Poetry as Performance: Homer and Beyond,Cambridge: Cambridge University Press.

Nagy, G. (2000) "Reading Greek Poetry Aloud. Evidence from the Bacchylides Papyri", QUCC,64, pp. 7-28.

Nagy, G. (2010), "Language and Meter", en E. J. Bakker (ed.) A Companion to the Ancient Greek Language, West Sussex: Wiley-Blackwell, pp. 370-387. 
O'Neill JR., E. G. (1942) "The Localization of Metrical Word Types in the Greek Hexameter", YClS, 8, pp. 103-178.

Porter, H. N. (1951) "The Early Greek Hexameter", YClS,12, pp. 1-63.

Probert, P. (2006) Ancient Greek Accentuation. Synchronic Patterns, Frequency Effects and Prehistory, Oxford: University Press.

SAUZET, P. (1989) "L'accent du grec ancien et les relations entre structure métrique et representationautosegmentale", Langages, 95, 81-113.

Silva BarRIS, J. (2011)Metre and Rhythm in Greek Verse, Wien: oaw.

Sommerstein, A. H. (1973)The Sound Pattern of Ancient Greek, Oxford: Basil Blackwell.

SteriAde, D. (1988) "Greek Accent: A Case for Preserving Structure", Linguistic Inquiry, 19, 271-314.

VendRYes, J. (1904) Traitéd'accentuationGrecque, Paris:Klincksieck.WeSt, M. L. (1982) Greek Metre, Oxford: University Press. 